\title{
Splay orientation in a capillary
}

\author{
Miłosz S. Chychłowski and Tomasz R. Woliński \\ Faculty of Physics, Warsaw University of Technology, Koszykowa 75, 00-662 Warszawa,
}

Received September 22, 2010; accepted December 17, 2010; published December 31, 2010

\begin{abstract}
The work presents a new type of orientation and method of liquid crystal alignment control inside a capillary. This method is a totally new approach which uses thermal activation to create an orienting polymer layer on the inner surface of a capillary. By using the polyamic acids SE-1211 that induce vertical anchoring, we obtained a splay orientation of the $6 \mathrm{CHBT}$ nematic liquid crystal in the capillary.
\end{abstract}

Photonic crystal fibers (PCFs) are the latest achievement in optical fiber technology [1]-[2]. PCFs are 2D-photonic crystals with a defect as a core that is surrounded by a cladding structure with a periodic refractive index change around the fiber core. Various materials such as pure and doped silica are used but in the majority of PCFs air holes play the role of a second material. This approach has a great advantage over any other material because after the manufacturing process of the fiber, these air holes can be later infiltrated by gases or fluids. To a special kind of fluids belong liquid crystals (LCs) that generally possess elongated anisotropic organic molecules. LCs, as mesomorphic materials, have the properties of both solid crystals such as high order of molecules arrangement and liquids that can relatively easily penetrate micro-scale holes, and their arrangement may be influenced by external factors [3]. PCFs filled with LCs create new possibilities in optical communication and sensing technology.

Photonic Liquid Crystal Fibers (PLCFs), as a combination of PCFs and LCs, create a great opportunity to make a new generation of advanced photonic devices [4]-[7]. The PCFs infiltrated with LCs gain additional properties due to the presence of a highly-tunable material. Consequently, light propagation in the PLCFs acquires new ways to tune its properties. LC molecules inside micro-scale tubes of a PCF can be reoriented by external fields as well as by changes in optical anisotropy. The use of an LC to dynamically change PCF propagation properties requires a high level of repeatability and stability at every state induced by external electric or magnetic fields. Both fields can easily create a stable molecule orientation, but without the fields, LC molecules reorient to minimize their free energy. In order to control LC alignment we need to use additional solutions, similar to these used in LC cells technology.

Typical diameters of air holes in PCFs are within the range of few to tens of micrometers, which is similar to the dimensions of LC cells commonly used.

Passive control of LC orientation in PCFs is one of the key issues while manufacturing a photonic device. Similarly to LC cells, surface anchoring plays a major role in LC molecules arrangement. The most common method of creating an anchoring layer for LC cells is rubbing, which is used due to easy access to the surface of the cells. This method as well as others requiring direct access to the orienting surface cannot be applied to the micro-scale tubes inside the PCFs and this is the reason why we have recently proposed a photoalignment method to induce planar or vertical orienting layers within the PCF microholes [8]-[10].

In this letter we present another method for indirect orienting layer creation, which is based on thermallyinduced activation of the orienting layer on the inner surface of PCF air tubes [11]. Thermally-induced creation of the aligning layer is a method that is based on polymerizing polyamic acids into polymer chains by heating them up to the required temperature. Vertical, planar, and/or tilted aligning layers are the results of this procedure.

In our experiment we used a capillary with a diameter of about 8 micrometers (manufactured at the Maria Curie Sklodowska University - UMCS Lublin, Poland) representing a single air tube. As an orienting material a SE-1211 (Nissan Chemical Industries, Ltd.) was used and 6CHBT nematic liquid crystal mixture (manufactured at the Military University of Technology - MUT, Warsaw, Poland) was selected. 
The preparation procedure of the sample involved filling the whole capillary with SE1211 under high pressure and then removing excess SE1211 by air under high pressure leaving only a thin layer. This stage takes usually from tens of minutes to a few days depending on the capillary length and its diameter. The next step was to polymerize the SE1211 mixture in the capillary by high temperature: the vertical alignment needs temperatures at the range $120-180$ Celsius for a few hours to polymerize. The last step was to fill the capillary with the LC by capillary forces or high pressure depending on the sample length.

The vertical anchoring at the inner surface of the capillary tube filled with a nematic LC resulted in the splay-type orientation (also known as axial orientation). The LC molecules are vertical to the surface (homeotropic alignment) due to the orienting layer and they are being continuously deformed to be parallel to the capillary axis in its central part (see Figs 1 and 2).

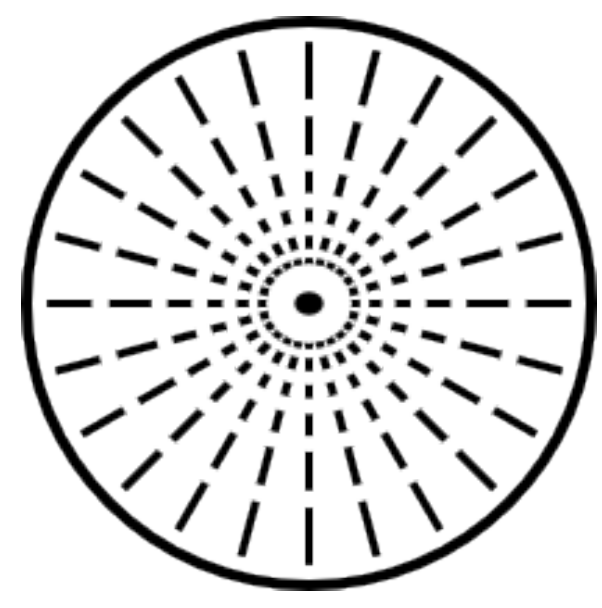

Fig. 1. Splay-deformed LC molecules: cross-section in the capillary.

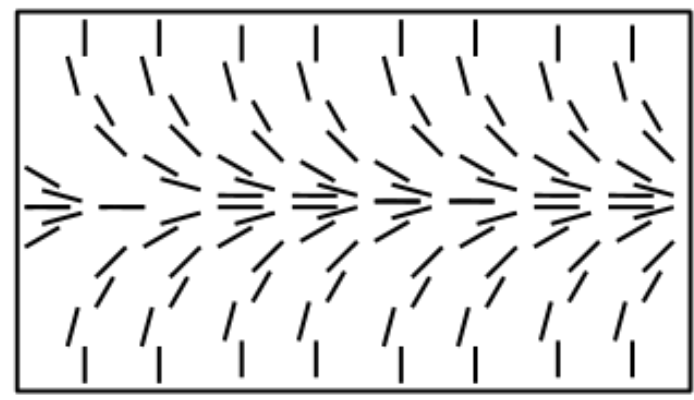

Fig. 2. Splay-deformed LC molecules in the capillary axis.
The prepared samples were observed under a polarizing microscope. Figure 3 a presents a situation in which both crossed polarizers' axes are either parallel or perpendicular to the capillary long axis. Due to the rotation of the sample (within the image plane), the axis of polarized light does not coincide with the fiber axis, and the birefringence of the splay-deformed sample would be responsible for the observed shift of the bright coloured patterns. These images confirm that inside a capillary there is a splay orientation.

We have also observed two changes (point defects) in Fig. 3. These changes are a consequence of the cooling process (from the isotropic phase) and can be attributed to disclinations points. As one can see, at each side of the defect point there is a change of the birefringence sign caused by a change in the LC direction flow.
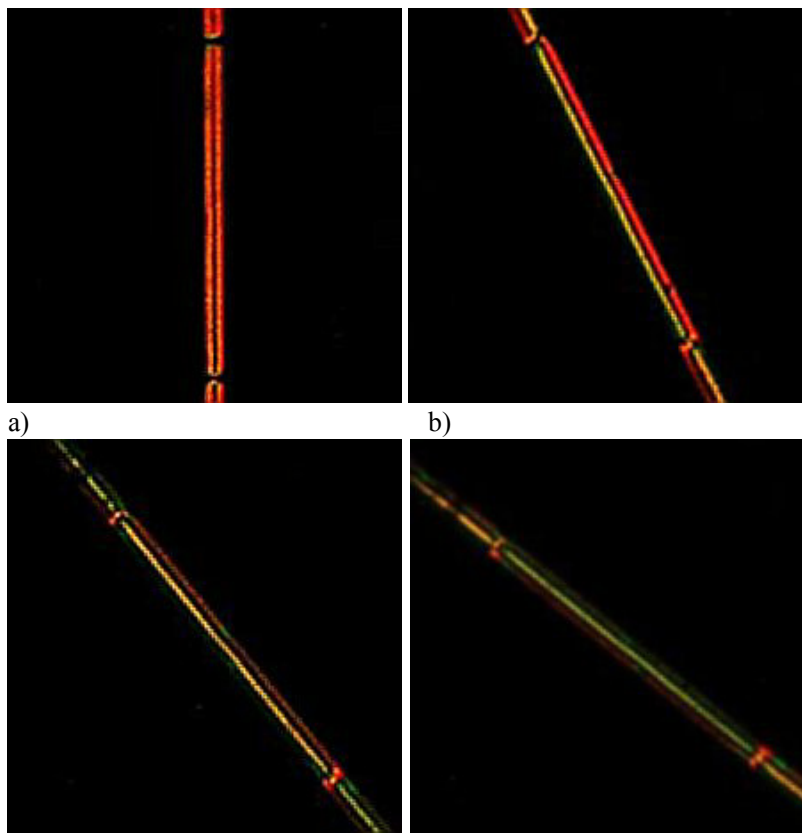

c)

d)

Fig. 3. Images of the capillary filled with splay-deformed 6CHBT under the polarizing microscope with crossed polarizers at a) 0 b) 20 c) 40 d) 60 degrees to polarizer axis.

In the paper, the new technique of LC orientation inside capillaries has been investigated. The manufactured orienting layer enabled to induce a splay-type LC configuration inside the capillary tube. This technique can greatly improve the manufacturing of photonic devices based on PLCF technology. 
This work was supported by the Polish Ministry of Science and Education under the grant N517 056535 and N507 439539. M. Chychłowski acknowledges the European Social Fund through the Warsaw University of Technology Development Programme granted by the European Union. This work has been also partially supported by the MISTRZ Programme of the Polish Science Foundation. The authors would like to thank E. Nowinowski-Kruszelnicki (MUT) for the orienting material.

\section{References}

[1] P. St. J. Russell, Science 299, 358 (2003).

[2] C. Knight, Nature 424, 847 (2003).

[3] H. Kawamoto, Proc. IEEE 90(4), 460 (2002).
[4] T. Woliński, A. Czapla, S. Ertman, M. Tefelska, A. Domański, J. Wójcik, E. Kruszelnicki, R. Dabrowski, IEEE Trans. Instrum. Meas. 57, 1796 (2008).

[5] T.R. Wolinski, S. Ertman, P. Lesiak, A.W. Domanski, A. Czapla, R. Dabrowski, E. Nowinowski-Kruszelnicki, J. Wojcik, OptoElectron. Rev. 14, 329 (2006).

[6] T. Nasiłowski, P. Lesiak, R. Kotyński, M.K Antkowiak, F. Berghmans, P. Mergo, J. Wójcik, H. Thienpont, Proc. SPIE 5576, 68 (2004).

[7] T.R. Woliński, S. Ertman, M. Tefelska, A. Czapla, D. Budaszewski, A.W. Domański, R. Dąbrowski, E. NowinowskiKruszelnicki, J. Wójcik, Mol. Cryst. Liq. Cryst. 489, 169 (2008).

[8] S. Ertman, T.R. Woliński, A. Czapla, K. Nowecka, E. Nowinowski-Kruszelnicki, J. Wójcik, Proc. SPIE 6587, 658706 (2007).

[9] M.S. Chychłowski, S. Ertman, T. R. Woliński, Phot. Letter of Poland 2(1), 28 (2010).

[10] V.V. Presnyakov, Z.J. Liu, V.G. Chigrinov, Proc. SPIE 6017, 60170J-1 (2005).

[11] K.E. Vaughn, M. Sousa, D. Kang, C. Rosenblatt, Appl. Phys. Lett. 90(19), 194102 (2007). 